\title{
Clinical and economic outcomes of treatment sequences for intermediate- to poor-risk advanced renal cell carcinoma
}

\author{
Apoorva Ambavane *,1, Shuo Yang², Michael B Atkins ${ }^{3}$, Sumati Rao ${ }^{2}$, Anshul Shah ${ }^{4}$, \\ Meredith M Regan ${ }^{5}$, David F McDermott ${ }^{6} \&$ M Dror Michaelson ${ }^{7}$ \\ ${ }^{1}$ Evidence Synthesis, Modeling, \& Communications, Evidera, Inc., Bethesda, MD 20814, USA \\ ${ }^{2}$ Health Economics Outcomes Research, Bristol-Myers Squibb, Princeton, NJ 08540, USA \\ ${ }^{3}$ Department of Internal Medicine - Medical Oncology, Georgetown-Lombardi Comprehensive Cancer Center, Washington, DC \\ 20007-2113, USA \\ ${ }^{4}$ Evidence Synthesis, Modeling, \& Communications, Evidera, Inc., Waltham, MA 20814, USA \\ ${ }^{5}$ Division of Biostatistics, Dana-Farber Cancer Institute, Harvard Medical School, Boston, MA 02215, USA \\ ${ }^{6}$ Department of Medicine, Beth Israel Deaconess Medical Center, Dana-Farber/Harvard Cancer Center, Boston, MA 02215, USA \\ ${ }^{7}$ Massachusetts General Hospital Cancer Center, Boston, MA 02214, USA \\ *Author for correspondence: apoorvaam@gmail.com (Apoorva Ambavane was an employee of Evidera at the time the analysis \\ was conducted)
}

Aim: To assess the cost-effectiveness of treatment sequences for patients with intermediate- to poor-risk advanced renal cell carcinoma. Patients \& methods: A discrete event simulation model was developed to estimate patients' lifetime costs and survival. Efficacy inputs were derived from the CheckMate 214 and CheckMate 025 studies and network meta-analyses. Safety and cost data were obtained from the published literature. Results: The estimated average quality-adjusted life-years (QALYs) gained was the highest on nivolumab + ipilimumab-initiated sequences (3.6-5.3 QALYs) versus tyrosine kinase inhibitor (TKI)-initiated sequences (2.1-3.7 QALYs). Incremental cost per QALY gained for nivolumab + ipilimumabinitiated sequences was below the willingness-to-pay threshold of $\$ 150,000$ versus other sequences. Conclusion: Immuno-oncology combination therapy followed by TKIs is cost-effective versus TKI sequences followed by immuno-oncology or sequencing TKIs.

First draft submitted: 7 November 2019; Accepted for publication: 17 December 2019; Published online: 29 January 2020

Keywords: cost-effectiveness $\bullet$ immuno-oncology therapy $\bullet$ renal cell carcinoma $\bullet$ survival $\bullet$ treatment sequences - treatment-free interval

Renal cell carcinoma (RCC) accounts for 90\% of all kidney cancer cases in the USA [1]. Advanced or metastatic RCC (aRCC) has a poor prognosis, with a 5-year relative survival rate of $12 \%$ reported for patients diagnosed between 2009 and 2015 in the USA [2,3]. Per International Metastatic Renal Cell Carcinoma Database Consortium (IMDC) classification, approximately $80 \%$ of patients with aRCC have intermediate- or poor-risk disease $[4,5]$ and historically have had a shorter survival duration compared with overall aRCC patients (median overall survival [OS] for treatment with molecular-targeted agents: overall, 41 months; intermediate risk, 33.6 months; poor risk, 15.2 months) [6]. The need for effective therapies is even greater in patients with IMDC risk stratification of intermediate or poor.

The treatment landscape of aRCC has evolved over the past two decades, initially with the introduction of targeted therapies and later with immuno-oncology therapies (I-Os). Guidelines include various drug classes such as tyrosine kinase inhibitors (TKIs); mammalian target of rapamycin inhibitors as monotherapy or in combination with lenvatinib; the monotherapy, nivolumab; and I-O combination therapies that include nivolumab + ipilimumab, axitinib + pembrolizumab and axitinib + avelumab [7]. The treatment pathway can comprise various sequences including, for example, I-O combination treatments followed by TKIs, TKIs followed by I-O or TKIs followed by TKIs [7]. Clinical trials often evaluate treatment options for either first- or second-line treatment, but not the specific effect of a given treatment sequence. There is limited evidence for optimal treatment sequencing with an 
increasing number of options becoming available $[8,9]$. With the availability of multiple drug classes, including mono- or combination therapies, there is a need to gain a deeper understanding of the optimal sequence for firstand second-line treatment of specific patient subsets.

The assessment of TKIs and I-Os requires special consideration of their mechanisms of action and impact on long-term outcomes. I-Os are associated with differences in response patterns and adverse event (AE) profiles compared with TKIs, which impact treatment selection decisions in clinical practice. Long-lasting antitumor responses can occur in patients who respond to I-Os during treatment, as well as in patients who have discontinued I-O treatment [10-15]. Durable responses beyond treatment cessation result in a long treatment-free interval (TFI; a measure of durability of response between treatment discontinuation and time to subsequent treatment) or a long treatment-free survival (TFS; a measure of durability of response between treatment discontinuation and time to subsequent treatment or death, whichever occurs first) and delay or even obviate the need for subsequent treatment. Although not identical, both TFI and TFS metrics characterize antitumor activity during the treatmentfree period that occurs after I-O therapy discontinuation. Patients who receive I-O treatment and then discontinue therapy often enter into a treatment-free period and, although not current standard strategy, it is a common enough occurrence to require its consideration in the sequence of treatments. Post hoc analyses of the CheckMate 214 trial (minimum follow-up of 30 months) showed that 24 months after treatment discontinuation, $19 \%$ of patients receiving nivolumab + ipilimumab did not require second-line treatment compared with $6 \%$ receiving sunitinib [16]. Furthermore, the median TFS was 9.6 months (95\% CI: 5.7 months-not evaluable) in patients who achieved a response on nivolumab + ipilimumab compared with 1.9 months (95\% CI: 1.5-3.1 months) for those responding to sunitinib [16]. Additionally, in an analysis of all CheckMate 214 patients (not just those who discontinued), nivolumab + ipilimumab provided longer survival and delayed time to subsequent therapy versus sunitinib, overall as well as among those with intermediate to poor risk [17]. It is of note, however, that TFI data have not been published for I-O-TKI combinations. Therefore, TFI is an important consideration for I-Os since it reflects a time period in which patients will not incur drug costs or experience treatment-related AEs and potentially have improved quality of life.

Additionally, I-Os may be associated with a heterogenous response pattern characterized by 'pseudo-progression,' due to infiltration of immune cells in the tumor showing increased tumor size followed by a sustained response [18]. Because this response pattern can be associated with improved survival, conventional response measures, such as progression-free survival (PFS), may not be appropriate to determine treatment failure on I-Os as with the targeted therapies [19-21]. Hence, an assessment of alternative potential surrogate end points, such as the combination of treatment duration and TFI, can be considered. TFI is well established in hematologic cancers and is known as an important prognostic factor and treatment-effect modifier [22,23]. This concept has also been observed in solid tumors, such as patients with metastatic melanoma who discontinued nivolumab + ipilimumab and experienced a longer TFI based on a patient-level simulation model using the CheckMate 067/069 trial data (mean time spent in TFI: nivolumab + ipilimumab, 5.3 years; nivolumab, 3.4 years; ipilimumab, 2.3 years) [24].

Simulation models can be used to estimate the health benefits and economic outcomes associated with treatment sequences cycling between various classes of drugs. Using published clinical trial data, health economic models can be used to extrapolate data over long-term periods to estimate clinical outcomes and cost consequences associated with various treatment sequences. Additionally, economic models can capture aRCC natural disease progression and the impact of treatments with various mechanisms of action.

\section{Patients \& methods}

Model overview

A patient-level discrete event simulation was developed to estimate cost and health outcomes (discounted at 3\% per annum) over patients' lifetimes for treatment of previously untreated, IMDC intermediate- or poor-risk adult ( $\geq 18$ years) patients with aRCC, from the perspective of US third-party payers. The time horizon for the model analysis was 40 years, which should provide sufficient time to capture outcomes over patients' lifetimes given the median baseline age of the CheckMate 214 trial population was 62 years [11] and assuming that patients do not survive beyond 100 years of age. This model simulates patient flow through various disease milestones and treatment sequences by incorporating detailed clinical trial data, patient characteristics and the correlation of clinical outcomes. Underlying patient flow through various treatment sequences was based on patient-level data from the Phase III CheckMate 214 (NCT02231749) and CheckMate 025 (NCT01668784) trials [10,11] and network meta-analyses (NMAs) using published clinical trial data for TKIs. 


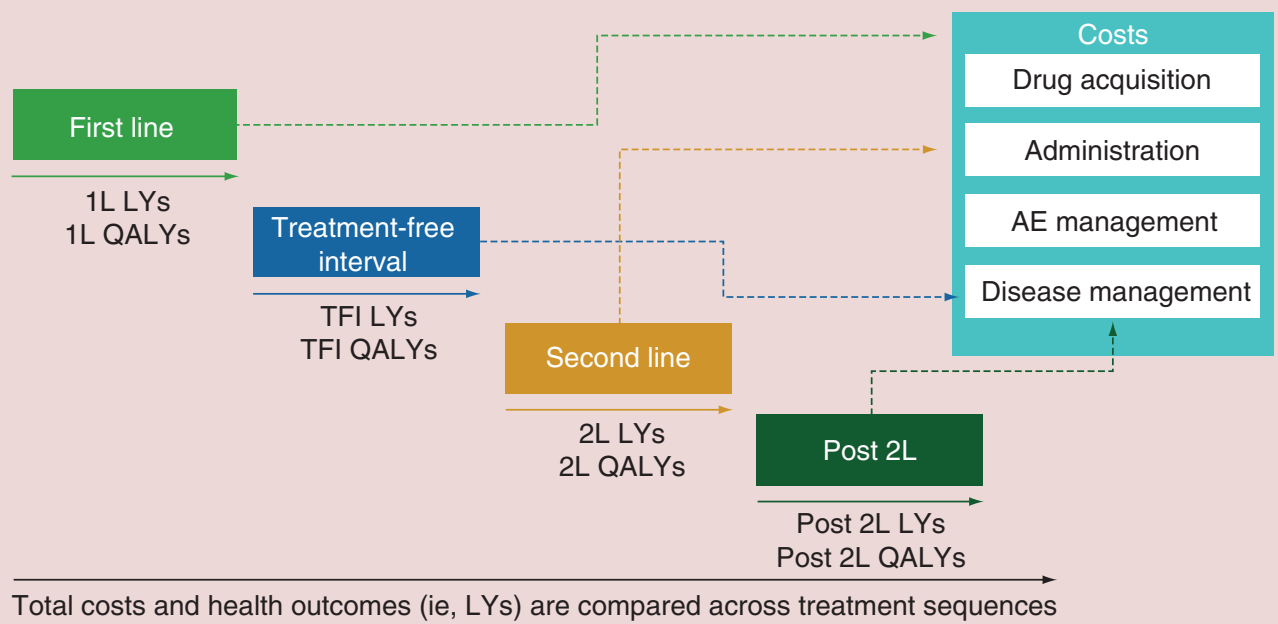

Figure 1. Model structure.

1L: First line; 2L: Second line; AE: Adverse event; LY: Life-year; QALY: Quality-adjusted life-year; TFI: Treatment-free interval.

\section{Treatment sequences}

Treatment options for management of aRCC were based on the National Comprehensive Cancer Network guidelines [7] and current US treatment paradigms, and finalized using clinical opinion. The sequences included are as follows:

- First-line nivolumab + ipilimumab followed by second-line cabozantinib, axitinib, pazopanib or lenvatinib + everolimus;

- First-line cabozantinib followed by second-line nivolumab, axitinib, pazopanib or lenvatinib + everolimus;

- First-line sunitinib or pazopanib followed by second-line nivolumab, cabozantinib, axitinib or lenvatinib + everolimus.

Although bevacizumab, sorafenib, everolimus, temsirolimus and sunitinib (as second-line treatment) are recommended by the National Comprehensive Cancer Network guidelines [7], their use in clinical practice in the USA is very limited. I-O + TKI combinations (pembrolizumab + axitinib and avelumab + axitinib) for treatment of aRCC, recently approved by the US FDA, were not included in this analysis as the published literature does not present details on clinical outcomes (such as Kaplan-Meier curves) for the intermediate- or poor-risk subpopulation, or TFI, and the follow-up time is too short to reliably model patients' long-term survival [25,26].

\section{Model structure}

The model simulated a cohort of patients with distinct baseline characteristics for each treatment sequence evaluated. Based on patient characteristics from CheckMate 214 (Supplementary Table 1) [11], the model predicted the time to clinical events for each line of treatment included in a sequence (Figure 1).

In the simulation, patients with aRCC initiate first-line treatment and can subsequently discontinue treatment due to AEs or disease progression. Upon discontinuation, patients may remain treatment-free for a certain period until the start of a second-line treatment or alternatively may never require a second-line treatment, which is defined as TFI. Upon discontinuation of second-line treatment, patients are assumed to receive the best supportive care until they experience death or reach the end of model time horizon. Patients can experience death in any phase of treatment.

For each treatment sequence, the time on first-line treatment, time between first- and second-line treatment (i.e., TFI), time on second-line treatment and time spent after second-line treatment discontinuation on the best supportive care are accrued over a patient's lifetime (over a 40-year time horizon; Figure 1). Drug acquisition, administration and AE management costs are accrued for the duration of each line of treatment. Disease manage- 
ment costs are accrued based on a patient's treatment status (on or off treatment) as a proxy for clinical benefit (progression-free or progressed) over lifetime.

\section{Efficacy inputs}

Patient profiles simulated

A cohort was simulated using individual patient profiles $(\mathrm{N}=779)$ from CheckMate 214 . In this simulation, the cohorts are cloned and are assigned a specific treatment sequence to ensure that the comparison is influenced by treatment and not by other patient characteristics. This is in contrast to the CheckMate 214 trial, where patients either received nivolumab + ipilimumab or sunitinib as first-line treatment and a mix of various second-line treatments. The purpose of this simulation is to estimate the cost of each treatment sequence based on the specific treatment sequence assigned by the simulation each time the cohort of patient profiles is run.

\section{Modeling of treatment sequences starting with nivolumab + ipilimumab, sunitinib \& pazopanib}

Multivariate risk equations for nivolumab + ipilimumab and sunitinib initiating sequences were derived using patient-level data from CheckMate 214 for the nivolumab + ipilimumab and sunitinib arms, respectively. Baseline patient characteristics of the study population are reported in 'Patient characteristics' in the Supplementary Information. The risk equations captured the impact of individual patient characteristics, past treatment history (e.g., radiotherapy), first- and second-line treatment and interim disease milestones (e.g., response, AE-related discontinuation, treatment duration or TFI) on long-term outcomes, such as OS. For each patient simulated in the model, the risk equations predicted best overall response, time to first-line treatment discontinuation (FLTD), time to second-line treatment initiation and competing risk of death in each treatment phase (i.e., time to death in the treatment-free interval phase and time to death after second-line treatment). The predictive validity of risk equations was established by comparing the model-simulated outcomes versus the observed data from CheckMate 214 [11]. Additional details on the derivation, implementation and validation of risk equations is provided in 'Model inputs: nivolumab + ipilimumab and sunitinib starting sequences' in the Supplementary Information.

Following nivolumab + ipilimumab, the impact of second-line treatment with axitinib and pazopanib on survival was based on CheckMate 214 data. Few patients received cabozantinib and lenvatinib + everolimus as second-line treatment in the CheckMate 214 population. Furthermore, following first-line sunitinib, the treatment effect of second-line treatments led to unreliable estimates compared with published randomized clinical trials. Due to insufficient data from CheckMate 214, the estimated hazard ratios (HRs) in a previously conducted NMA for cabozantinib and lenvatinib + everolimus were applied to the risk of death after second-line initiation on sunitinib (see 'Overall survival on second-line treatments' in the Supplementary Information).

Treatment sequences starting with pazopanib were modeled using the risk equations for sunitinib. This was based on the results of the COMPARZ trial, which reported similar efficacy for sunitinib and pazopanib. HR for death with pazopanib versus sunitinib was 0.91 (95\% CI: 0.76-1.08; $\mathrm{p}=0.28)$ [27].

\section{Modeling of treatment sequences starting with cabozantinib}

Given the absence of clinical trials providing head-to-head comparison between cabozantinib and nivolumab + ipilimumab in the first-line setting, an NMA was conducted [28]. This analysis estimated the treatment effect of cabozantinib versus sunitinib using data from CABOSUN [29] and CheckMate 214 [11]. The estimated HRs for first-line treatment with cabozantinib are 0.66 (95\% CI: 0.46-0.95) for PFS and 0.80 (95\% CI: 0.50-1.27) for OS versus sunitinib [30]. Since progression is the most common reason for treatment discontinuation, we assume that the treatment effect for progression is applicable to treatment discontinuation. The NMA-based HRs were applied to the model and predicted FLTD and OS for sunitinib (see 'Model inputs: cabozantinib starting sequences' in the Supplementary Information), respectively.

There is no published evidence to inform the duration of TFI after treatment with cabozantinib. The risk equation for TFI and competing risk of death in TFI for sunitinib were applied using interim disease milestones on cabozantinib (e.g., treatment duration, best overall response and AE-related discontinuation) as predictors.

\section{Modeling of treatment duration on second line}

Limited data were available in CheckMate 214 on second-line treatment duration as most patients initiating second-line treatment did not have their date of discontinuation recorded [11]. Parametric distributions were fitted to Kaplan-Meier curves for time to treatment discontinuation on everolimus from CheckMate 025 [10]. For other 


\begin{tabular}{|c|c|c|c|c|c|c|c|}
\hline & $\mathrm{NIVO}+\mathrm{IPI}^{\dagger}$ & SUN & AXI & PAZ & CAB & NIVO & LEN + EVE \\
\hline Drug cost/month & $\begin{array}{l}\$ 32,485 \text { (induction) } \\
\$ 13,887 \text { (maintenance) }\end{array}$ & $\$ 12,950$ & $\$ 15,302$ & $\$ 13,269$ & $\$ 18,633$ & $\$ 13,887$ & $\$ 33,885$ \\
\hline Administration cost/month & $\begin{array}{l}\$ 516 \text { (induction) } \\
\$ 647 \text { (maintenance) }\end{array}$ & $\$ 0^{\ddagger}$ & $\$ 0^{\ddagger}$ & $\$ 0^{\ddagger}$ & $\$ 0^{\ddagger}$ & $\$ 647$ & $\$ 0^{\ddagger}$ \\
\hline \multicolumn{8}{|c|}{ AE management over treatment duration } \\
\hline First line & $\$ 1868$ & $\$ 5138$ & NA & $\$ 6205$ & $\$ 6466$ & NA & NA \\
\hline Second line & NA & NA & $\$ 7835$ & $\$ 3945$ & $\$ 7415$ & $\$ 811$ & $\$ 8439$ \\
\hline \multicolumn{8}{|l|}{ Mean AE disutility/month } \\
\hline First line & -0.001 & -0.002 & NA & -0.003 & -0.003 & NA & NA \\
\hline Second line & NA & NA & -0.004 & -0.002 & -0.004 & -0.0003 & -0.004 \\
\hline Treatment phase & \multicolumn{4}{|c|}{ Disease management cost/month ${ }^{\S}$} & \multicolumn{3}{|c|}{ Utilities } \\
\hline First line & \multicolumn{4}{|c|}{$\$ 73.17$} & \multirow{2}{*}{\multicolumn{3}{|c|}{$\begin{array}{c}\text { NIVO + IPI: } 0.83 \\
\text { CAB, SUN, PAZ: } 0.79\end{array}$}} \\
\hline TFI & \multicolumn{4}{|c|}{$\$ 122.71$} & & & \\
\hline Second line & \multicolumn{4}{|c|}{$\$ 73.17$} & \multicolumn{3}{|c|}{ All therapies: 0.69} \\
\hline Post second line & & $\$ 122.71$ & & & \multicolumn{3}{|c|}{ All therapies: 0.61} \\
\hline \multicolumn{8}{|c|}{$\begin{array}{l}\text { All costs are reported in } 2018 \text { US dollar values. } \\
\dagger \text { Induction duration of } 2.76 \text { months ( } 0.23 \text { years). } \\
\ddagger \text { Assumed no costs associated with oral administration. } \\
\text { § Progression-free and progressed disease resources were assumed to be applicable for on-treatment (first line, second line, post second line) and off-treatment (TFI), respectively. } \\
\text { AE: Adverse event; AXI: Axitinib; CAB: Cabozantinib; EVE: Everolimus; IPI: Ipilimumab; LEN: Lenvatinib; NA: Not applicable; NIVO: Nivolumab; PAZ: Pazopanib; SUN: Sunitinib; TFI: } \\
\text { Treatment-free interval. }\end{array}$} \\
\hline
\end{tabular}

second-line treatments, HR for PFS from a previously conducted NMA was applied to the model-simulated time to treatment discontinuation on everolimus in CheckMate 025 (see 'Time to treatment discontinuation on second-line treatments' in the Supplementary Information) [29].

\section{Background mortality}

OS data reported in CheckMate 214 reported all-cause mortality (i.e., aRCC and non-aRCC related) [11]. Extrapolation of OS from the trial results showed deceleration of mortality hazards over a long-term period (see 'Model-predicted curves for key clinical outcomes' in the Supplementary Information). Hence, extrapolation purely based on trial data will likely overestimate OS. Given the mean age of 61 years for the intermediate- or poor-risk patient population in CheckMate 214, the hazard of death among the general US population [31] was observed to exceed that of the trial-based extrapolations at 22 years from the model start (i.e., at about age 83 years; see 'Comparison of general mortality vs trial-based mortality' in the Supplementary Information). As a conservative approach for OS estimation on aRCC treatments, the risk of all-cause death was applied to all patients after 22 years of model time horizon.

\section{Cost inputs}

Drug acquisition and administration costs per month were calculated using the drugs' wholesale acquisition cost, route of administration, unit costs for administration (payer reimbursement for intravenous drug administration in hospital outpatient setting), recommended dose and dosing frequency based on publicly available sources, FDA labels and clinical trials (Table 1; Supplementary Tables 9-11). The analysis included only grade 3 and 4 AEs due to their high cost burden. Drug-specific AE incidence was taken from the published clinical trials and the corresponding cost of inpatient management was obtained from the published literature (Table 1; Supplementary Tables 12 \& 13). Treatment-related costs were applied for the entire duration of treatment except for nivolumab + ipilimumab. A maximum treatment duration of 2 years was used, which is in line with maximum treatment duration assumed for previous economic evaluations of pembrolizumab and nivolumab monotherapy. The impact of maximum treatment duration was considered only for cost application and was not applied to efficacy outcomes in order to align model predictions with observed clinical data.

Annual rates of resource use associated with disease management (for being on-treatment or treatment-free) were obtained from published economic evaluations in patients with aRCC in the USA. Unit costs for resource use 
associated with the management of the disease were obtained from published sources (Table $1 \&$ Supplementary Table 14).

Costs not reported in the 2018 USD values were inflated to 2018 values using the medical care component of the US consumer price index [32].

\section{Utilities}

Utility index scores were derived from the previously published economic evaluation of aRCC treatments in first-line and second-line settings. Most of these publications reported utility values based on progression status (i.e., progression-free or progressive disease). Since this analysis evaluates time on each treatment phase, rather than progression status, we assumed time to next treatment (i.e., first-line treatment + TFI) as a proxy for clinical benefit and applied utilities reported for progression-free state for first-line treatments to these treatment phases. The model considered utility values for different treatment phases: first-line treatment $(0.83$ on nivolumab + ipilimumab and 0.79 on cabozantinib, sunitinib and pazopanib), TFI (same as first-line treatment), second-line treatment ( 0.69 on all therapies) and post second-line treatment ( 0.61 on all therapies). The utility index score on first-line treatment was estimated from responses to the EuroQoL 5-Dimensions instrument in CheckMate 214 [28]. Similarly, utility index scores for second-line treatment and post second-line treatment were estimated from responses to the EuroQoL 5-Dimensions instrument in the AXIS trial [33].

AE-related disutilities were derived from Swinburn $e$ t al. in patients with metastatic RCC [34]. Events with values not reported in Swinburn et al. were assigned an average disutility of other AEs (Supplementary Table 15). For all events, a duration of 10 weeks was applied for resolution as observed in CheckMate 214 [35].

\section{Model analyses}

The model estimated total life-years (LYs), quality-adjusted life-years (QALYs) and total costs over the model time horizon and calculated incremental cost per QALY gained for each treatment sequence. An efficiency frontier was created by plotting the total cost (horizontal axis) and QALYs (vertical axis) of each treatment sequence and drawing a line linking treatments that were not dominated by any of the other treatments in consideration.

Univariate and probabilistic sensitivity analyses were conducted to assess parameter uncertainty by varying parameter values as per the standard guidelines by the International Society for Pharmacoeconomics and Outcomes Research-Society for Medical Decision Making task force [36]. Probabilistic analyses, based on 1000 Monte Carlo simulations, were reported as cost-effectiveness acceptability curves to assess the impact of parameter uncertainty on the probability of individual sequences being the most cost-effective strategy over a range of willingness-topay (WTP) thresholds. The efficacy parameters were varied using multivariate normal distribution with variancecovariance matrix, HRs with lognormal distribution using their $95 \% \mathrm{CIs}$, safety parameters using beta distributions, and cost parameters using gamma distributions assuming $20 \%$ standard error, and utility parameters using beta distribution assuming 5\% standard error. A scenario analysis was conducted by assuming a 5-year maximum treatment duration for nivolumab + ipilimumab.

\section{Results}

The model estimated QALYs, LYs and costs of treatment sequences, which are shown in Supplementary Tables 16 and 17.

Drug acquisition costs were a key driver of total lifetime costs across all treatment sequences (Figures $2 \& 3$; Supplementary Tables $16 \&$ 17). First-line treatment costs accounted for approximately $34-83 \%$ of total costs among all sequences except sequences with lenvatinib + everolimus as second-line treatment, where second-line costs accounted for more than half (51-65\%) of total costs. The total costs on cabozantinib-initiating treatment sequences were higher than nivolumab + ipilimumab, sunitinib and pazopanib-initiating sequences (Figure 3).

Nivolumab + ipilimumab-initiating sequences were associated with the highest LYs (Figures $2 \& 4$ ) and QALYs (Supplementary Tables 16 \& 17; 4.75-7.60 LYs; 3.57-5.34 QALYs), followed by cabozantinib-initiating sequences (4.57-5.55 LYs; 3.14-3.76 QALYs), and then sunitinib/pazopanib-initiating sequences (3.11-4.24 LYs; 1.96-2.85 QALYs). The TFI was the longest with nivolumab + ipilimumab-starting sequences (1.25 LYs; 1.04 QALYs) compared with sequences starting with TKIs (0.47-0.78 LYs; 0.37-0.62 QALYs).

Nivolumab + ipilimumab-initiating sequences followed by axitinib, pazopanib and cabozantinib had the lowest total cost per LY (total lifetime costs divided by total LYs estimated) among all sequences analyzed (\$47,955/LY, $\$ 54,781 / \mathrm{LY}$ and $\$ 51,975 / \mathrm{LY}$, respectively; Figure 5). Nivolumab + ipilimumab followed by lenva- 


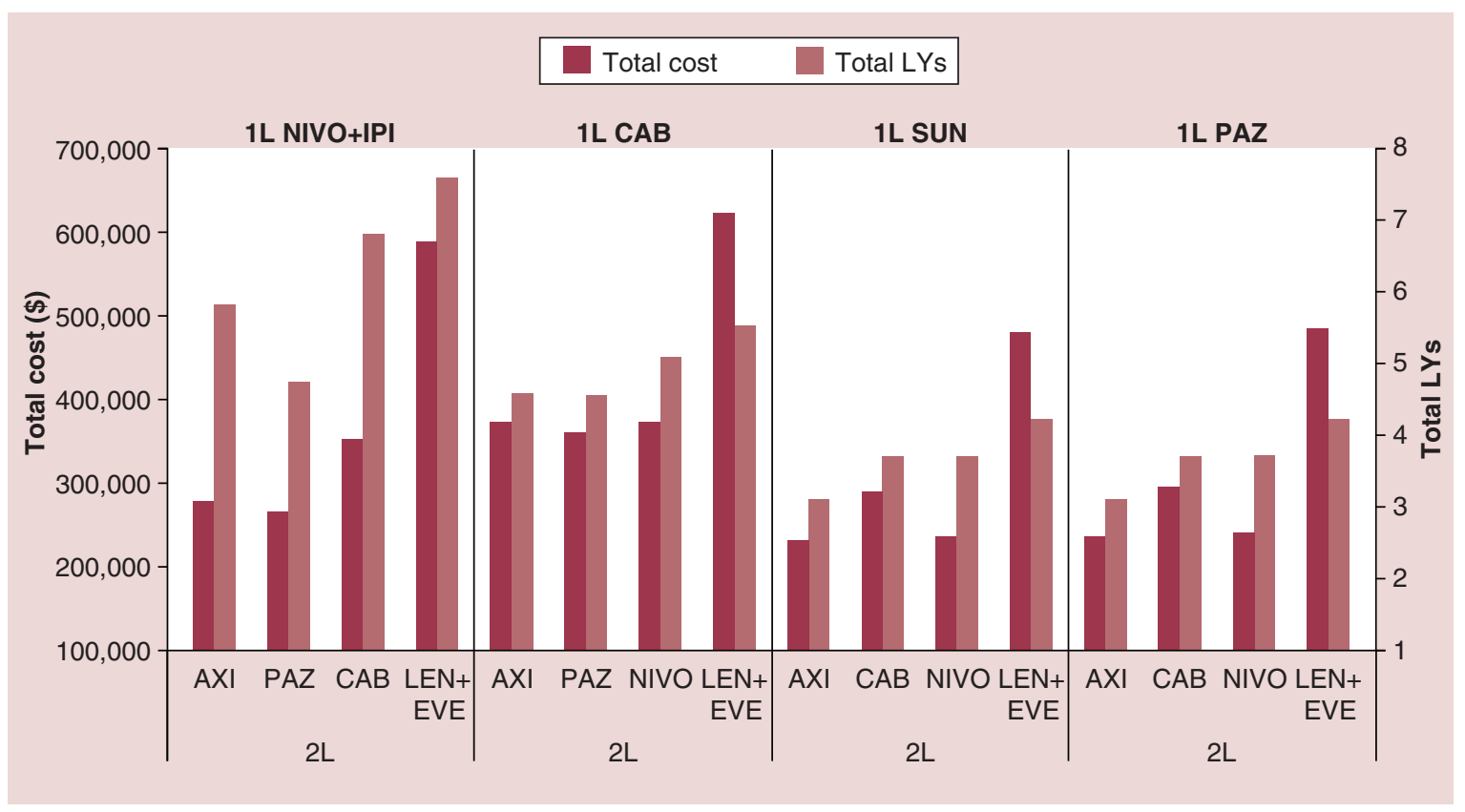

Figure 2. Total lifetime cost and life-years gained.

1L: First line; 2L: Second line; AXI: Axitinib; CAB: Cabozantinib; EVE: Everolimus; IPI: Ipilimumab; LEN: Lenvatinib; LY: Life-year; NIVO: Nivolumab; PAZ: Pazopanib; SUN: Sunitinib.

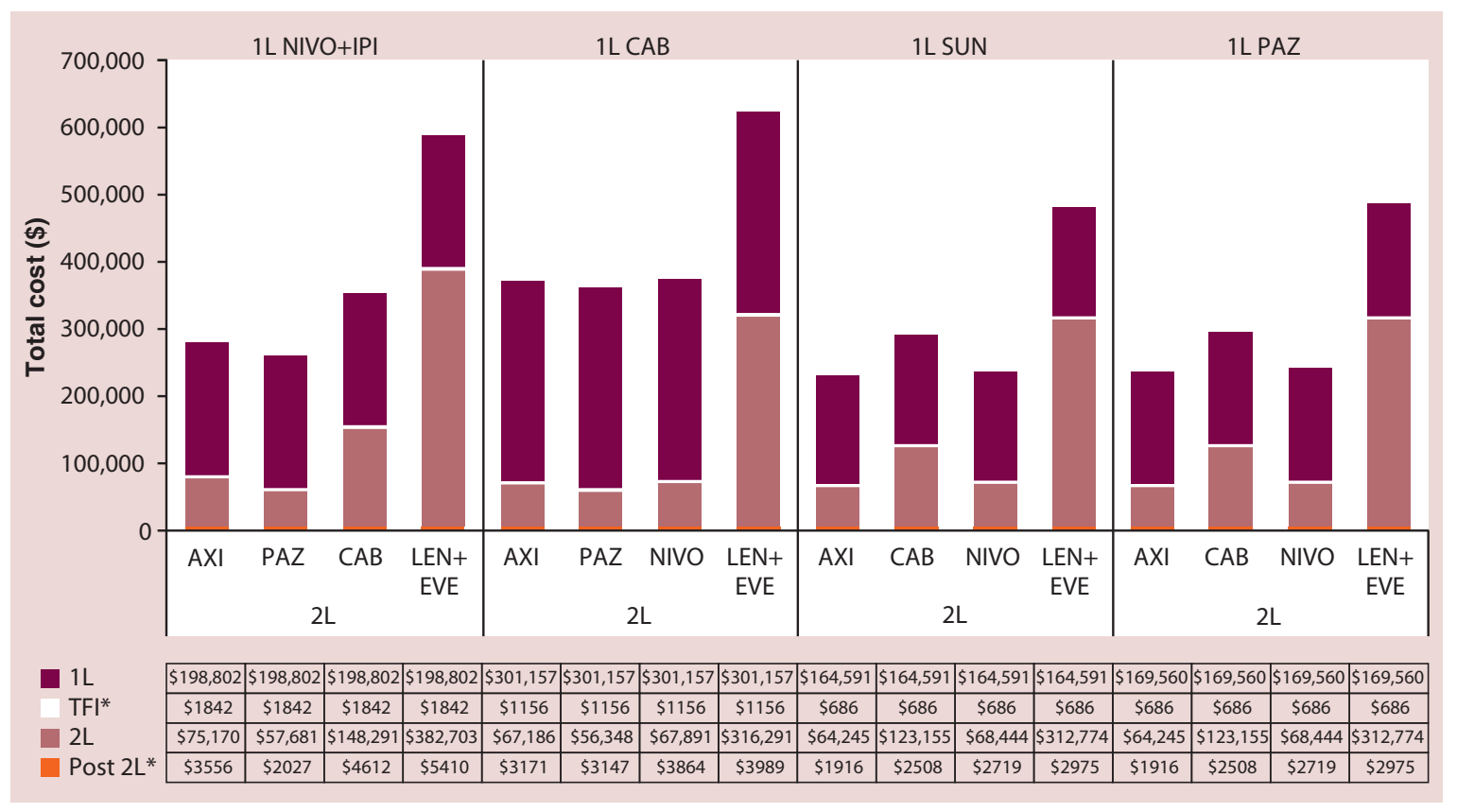

Figure 3. Costs associated with treatment sequences by treatment phase.

* See sub-table for the values for each bar.

1L: First line; 2L: Second line; AXI: Axitinib; CAB: Cabozantinib; EVE: Everolimus; IPI: Ipilimumab; LEN: Lenvatinib; NIVO: Nivolumab; PAZ: Pazopanib; SUN: Sunitinib; TFI: Treatment-free interval.

tinib + everolimus had an incremental cost-effectiveness ratio (ICER) of $\$ 77,421$ per LY due to higher drug costs associated with TKI combination treatment. 


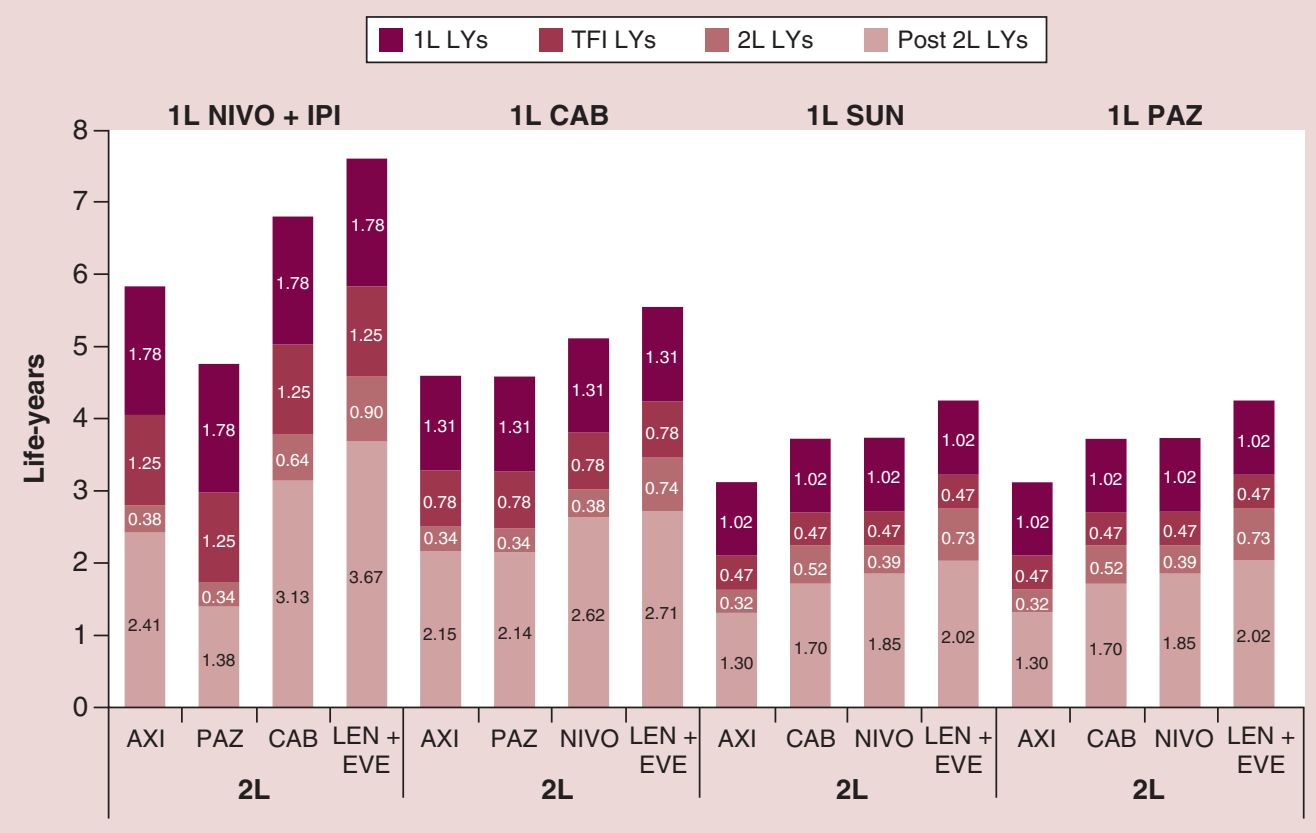

Figure 4. Life-years associated with treatment sequences by treatment phase.

1L: First line; 2L: Second line; AXI: Axitinib; CAB: Cabozantinib; EVE: Everolimus; IPI: Ipilimumab; LEN: Lenvatinib; LY: Life-year; NIVO: Nivolumab; PAZ: Pazopanib; SUN: Sunitinib.

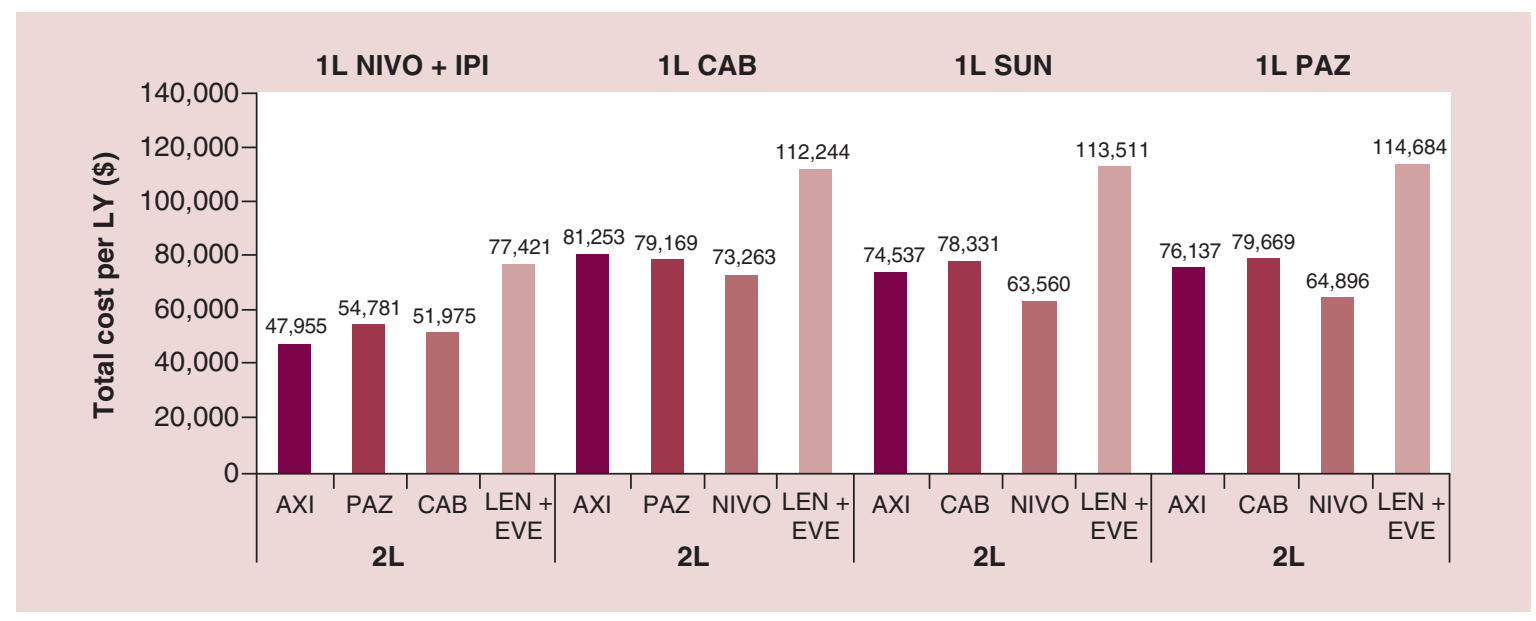

Figure 5. Total cost per life-year.

1L: First line; 2L: Second line; AXI: Axitinib; CAB: Cabozantinib; EVE: Everolimus; IPI: Ipilimumab; LEN: Lenvatinib; LY: Life-year; NIVO: Nivolumab; PAZ: Pazopanib; SUN: Sunitinib.

In terms of costs per QALY gained, nivolumab + ipilimumab followed by axitinib, pazopanib and cabozantinib sequences was associated with the lowest ICER among all sequences assessed $(\$ 66,357, \$ 72,927$ and $\$ 73,237$, respectively; Figure 6). In a pairwise comparison, sunitinib, cabozantinib and pazopanib sequences were either dominated (i.e., more costly and less effective) by nivolumab + ipilimumab-initiating sequences or had an ICER (calculated as costs per incremental QALY gained) lower than the WTP threshold of $\$ 150,000$ (ICER range of $\$ 16,524-\$ 125,860$; Table 2).

The univariate sensitivity analysis demonstrated that LYs and QALYs were the most sensitive to the parameters of the risk equation for OS on second-line treatment, HR of OS for cabozantinib versus sunitinib, parameters of the risk equation for FLTD on sunitinib, and total costs were most sensitive to drug costs and parameters of the 


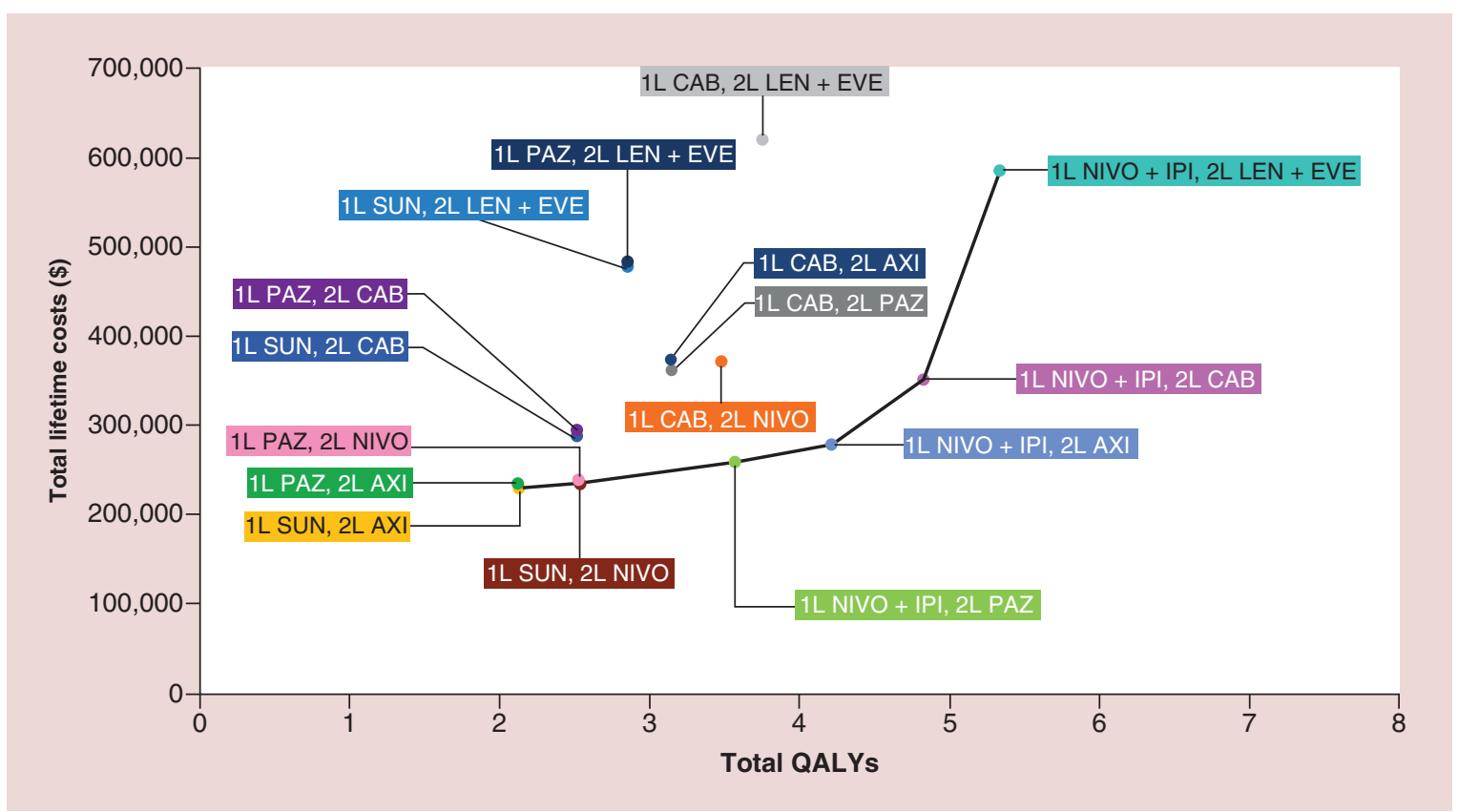

Figure 6. Efficiency frontier comparing treatment sequences for patients with intermediate- or poor-risk advanced renal cell carcinoma.

1L: First line; 2L: Second line; AXI: Axitinib; CAB: Cabozantinib; EVE: Everolimus; IPI: Ipilimumab; LEN: Lenvatinib; LY: Life-year; NIVO: Nivolumab; PAZ: Pazopanib; QALY: Quality-adjusted life-year; SUN: Sunitinib.

Table 2. Incremental costs per incremental quality-adjusted life-year gained for various treatment sequences in patients with intermediate- or poor-risk advanced renal cell carcinoma.

\begin{tabular}{|c|c|c|c|c|}
\hline $1 \mathrm{~L}$ & NIVO + IPI & NIVO + IPI & NIVO + IPI & NIVO + IPI \\
\hline $2 \mathrm{~L}$ & $\mathrm{AXI}$ & PAZ & $\mathrm{CAB}$ & LEN + EVE \\
\hline 1L CAB, $2 \mathrm{~L} \mathrm{AXI}$ & Dominated & Dominated & Dominated & $\$ 98,301$ \\
\hline 1L CAB, $2 \mathrm{~L} \mathrm{PAZ}$ & Dominated & Dominated & Dominated & $\$ 103,575$ \\
\hline 1L CAB, $2 \mathrm{~L}$ NIVO & Dominated & Dominated & Dominated & $\$ 116,004$ \\
\hline $1 \mathrm{~L} \mathrm{CAB}, 2 \mathrm{~L}$ LEN + EVE & Dominated & $\$ 1,949,084$ & Dominated & Dominated \\
\hline 1L SUN, 2L AXI & $\$ 23,019$ & $\$ 20,049$ & $\$ 45,233$ & $\$ 111,360$ \\
\hline 1L SUN, $2 \mathrm{~L}$ CAB & Dominated & Dominated & $\$ 27,095$ & $\$ 105,619$ \\
\hline 1L SUN, 2L NIVO & $\$ 25,663$ & $\$ 23,154$ & $\$ 51,135$ & $\$ 125,860$ \\
\hline 1L SUN, 2 L LEN + EVE & Dominated & Dominated & Dominated & $\$ 43,347$ \\
\hline 1L PAZ, 2L AXI & $\$ 20,564$ & $\$ 16,524$ & $\$ 43,281$ & $\$ 109,574$ \\
\hline 1L PAZ, $2 \mathrm{~L} \mathrm{CAB}$ & Dominated & Dominated & $\$ 24,870$ & $\$ 103,602$ \\
\hline 1L PAZ, 2L NIVO & $\$ 22,599$ & $\$ 18,220$ & $\$ 48,817$ & $\$ 123,778$ \\
\hline 1L PAZ, 2L LEN + EVE & Dominated & Dominated & Dominated & $\$ 41,233$ \\
\hline
\end{tabular}

risk equation for FLTD on sunitinib (Supplementary Figure 8). A cost-effectiveness acceptability curve (Figure 7) based on probabilistic sensitivity analyses showed that for a WTP threshold of $\$ 150,000$ per QALY gained, nivolumab + ipilimumab followed by axitinib and cabozantinib had the highest probability of being cost-effective. At higher WTP thresholds, first-line nivolumab + ipilimumab followed by lenvatinib + everolimus in secondline sequence becomes the most likely option to be cost-effective with a likelihood of approximately $30-85 \%$. Model results were robust to the probabilistic analyses, as deterministic and probabilistic means were comparable (Supplementary Table 18). Increasing maximum treatment duration of nivolumab + ipilimumab to 5 years resulted 


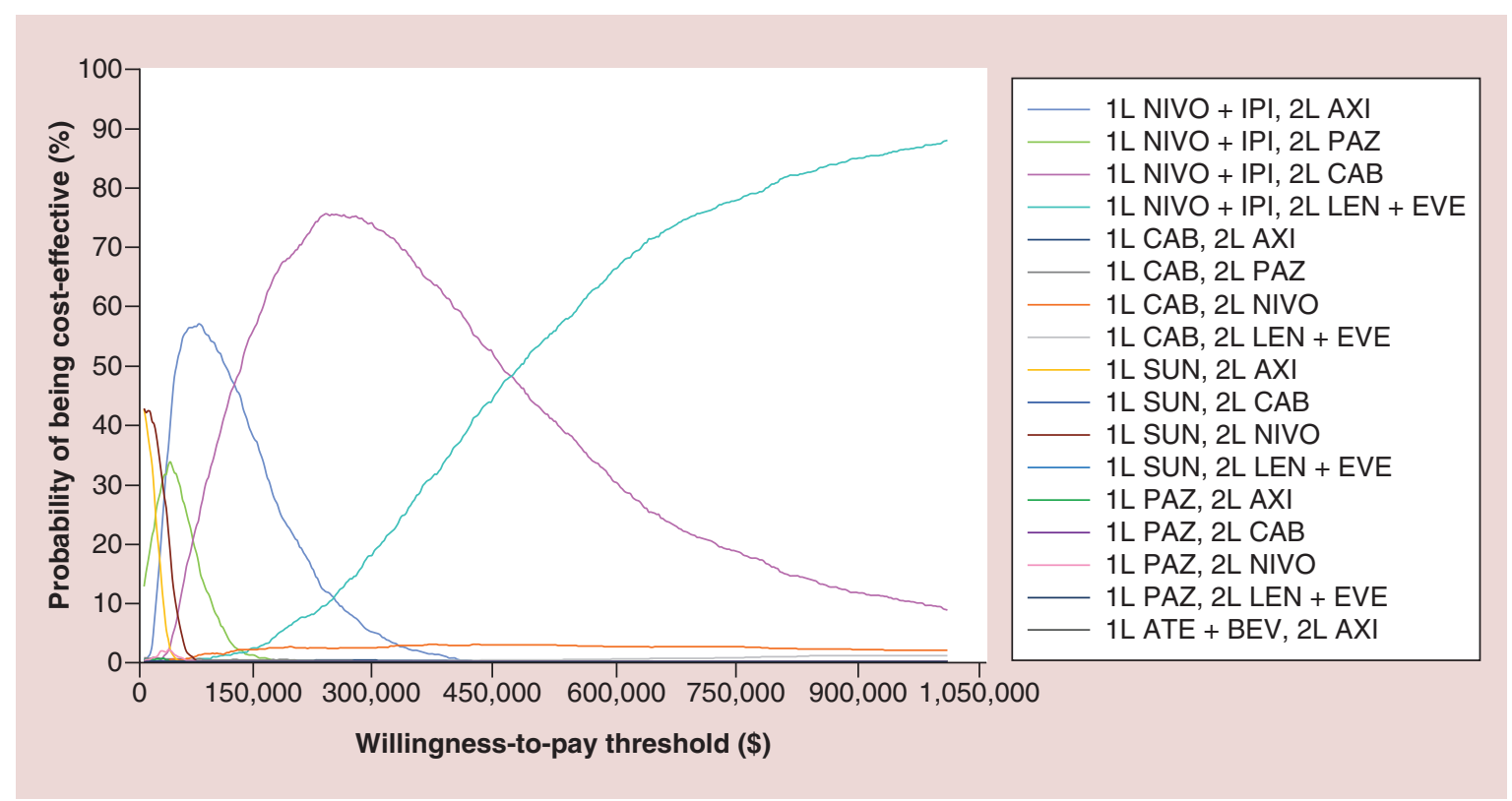

Figure 7. Cost-effectiveness acceptability curve for patients with intermediate- or poor-risk advanced renal cell carcinoma.

1L: First line; 2L: Second line; ATE: Atezolizumab; AXI: Axitinib; CAB: Cabozantinib; EVE: Everolimus; IPI: Ipilimumab; LEN: Lenvatinib; NIVO: Nivolumab; PAZ: Pazopanib; SUN: Sunitinib.

in the total cost per QALY (total lifetime costs divided by total QALYs estimated) of $\$ 82,641, \$ 92,129, \$ 87,390$ and $\$ 123,091$ on first-line nivolumab + ipilimumab followed by second-line axitinib, pazopanib, cabozantinib, and lenvatinib + everolimus sequences, respectively (Supplementary Table 19), and were lower compared with other sequences in the base case.

\section{Discussion}

The economic model was developed as a de novo simulation to estimate the cost and health outcomes associated with the first-line (nivolumab + ipilimumab, sunitinib, pazopanib, cabozantinib) and second-line (axitinib, pazopanib, cabozantinib, nivolumab, lenvatinib + everolimus) treatments commonly used in 2018 for IMDC intermediateor poor-risk patients with aRCC. To the best of our knowledge, this is the first study to evaluate cost and health outcomes of the 16 possible treatment sequences and their cost-effectiveness in aRCC.

The model predicted the highest LYs gained on the sequences with nivolumab + ipilimumab as a first-line treatment, which translated into a survival gain of 1-4 years (and corresponding gain of $0.46-3.21$ QALYs) compared with sequences with cabozantinib, sunitinib or pazopanib as a first-line treatment. The additional survival gain was mainly driven by the higher LYs gained on the first-line treatment, with two- to three-times higher TFI observed on nivolumab + ipilimumab sequences compared with other sequences. Notwithstanding the higher drug acquisition costs associated with nivolumab + ipilimumab, total costs per LY gained were lowest on nivolumab + ipilimumab followed by axitinib, pazopanib and cabozantinib sequences among all sequences analyzed. Consequently, ICER calculated as incremental costs per QALY gained were lowest for nivolumab + ipilimumab followed by axitinib, pazopanib and cabozantinib sequences $(\$ 66,357, \$ 72,927$ and $\$ 73,237$, respectively). The results were also consistent when a maximum treatment duration of 5 years was applied for nivolumab + ipilimumab treatment sequences (vs 2 years in the base case) and across different lengths of time horizons (10, 20 and 30 years; data not presented).

The simulation approach was undertaken in the absence of clinical trials evaluating sequential use of treatments as well as to model intricate risk equations and their correlations with patient characteristics and disease milestones. The key strength of the analyses is the detailed efficacy modeling nivolumab + ipilimumab and sunitinib sequences. Risk equations using patient-level data from CheckMate 214 were estimated to understand the impact of patient characteristics, disease characteristics, past and current treatments, and diseases milestones on clinical outcomes. Patients were tracked through their experience on first-line treatment, TFI, second-line treatment and post second- 
line treatment. Furthermore, the risk equations considered the impact of AE-related discontinuation and best overall response on clinical outcomes. The granularity of risk equations allowed us to accurately estimate the survival of patients given their profile and intermediate disease milestones. This approach enabled us to assess the impact of efficacy on I-O-TKI and TKI-TKI sequencing, given that second-line treatment agents are studied only for the patient population who previously had received sunitinib or pazopanib. The CheckMate 214 data allowed us to understand the interaction between first- and second-line treatments, specifically how survival would differ on second-line treatments, given past experience with nivolumab + ipilimumab or sunitinib. Nivolumab + ipilimumab as first-line treatment has an improved impact on survival after second-line initiation with TKIs compared with sunitinib. These findings aimed to provide important context in the decision of selecting the most efficient treatment sequence in patients with aRCC.

This study quantifies TFI in patients with aRCC, which has not been documented before. TFI as a clinical outcome has been established in hematologic cancers. Studies have also highlighted the experience of clinical benefit beyond treatment discontinuation with I-Os $[12,13,15]$. TFI is considered in metastatic melanoma, given the treatment benefit beyond cessation and pause in drug costs and toxicity. This analysis explored the TFI observed on nivolumab + ipilimumab and sunitinib by developing risk equation time in TFI. Over a patient's lifetime, nivolumab + ipilimumab was associated with an average of 0.8 years of TFI compared with 0.4 with cabozantinib and 0.3 years with sunitinib (Supplementary Figure 4). Given sunitinib and cabozantinib are TKIs, the observation of TFI was not expected. However, the risk equations showed that experiencing AEs (resulting in discontinuation) and poor performance scores were strong predictors for TKIs highlighting TFI on sunitinib, due to the time required for resolution of AEs and because poor performance status results in a delay or even omission of secondline treatment. In contrast, achieving response and higher IMDC risk scores were associated with longer TFI in nivolumab + ipilimumab, demonstrating that TFI on nivolumab + ipilimumab reflected response and clinical benefit beyond treatment discontinuation.

Our findings are comparable with the recently published economic evaluations in the USA [37-39] that compared nivolumab + ipilimumab using cohort-based models. One of the US analyses, Wan et al., compared nivolumab + ipilimumab and sunitinib in first-line setting over lifetime horizon [37]. The reported health benefits on nivolumab + ipilimumab and sunitinib were 3.99 LYs (2.84 QALYs) and 2.72 LYs (1.88 QALYs), respectively [37]. This was comparable to health gains on nivolumab + ipilimumab-starting sequences (4.75-7.60 LYs; 3.57-5.34 QALYs) and sunitinib-starting sequences (3.11-4.24 LYs; 2.13-2.85 QALYs). The reported ICER per QALY was $\$ 108,363 /$ QALY for nivolumab + ipilimumab versus sunitinib [37]. These results were within the ICER per QALY range reported on the nivolumab + ipilimumab-initiating sequences (dominated to $\$ 125,860 /$ QALY) in the current analysis. The other two US evaluations also compared nivolumab + ipilimumab and sunitinib in the first-line setting $[38,39]$. The ICER per QALY reported in these publications for nivolumab + ipilimumab versus sunitinib was $\$ 125,739 / \mathrm{QALY}$ [39] and $\$ 85,506 / \mathrm{QALY}$ [38] over a 10-year time horizon. This short time horizon made it difficult to directly compare with our cost-effectiveness result. However, it should be noted that none of the above-mentioned published economic evaluations have assessed the impact of second-line treatment on OS separately from first-line treatment [37-39]. The differences in ICERs can be explained by the cohort-based modeling approach used in these publications, which did not use patient-level data, versus our approach of considering individual patient characteristics, interim disease milestones and past as well as current treatment history [37-39]. Also, the published evaluations used PFS for treatment discontinuation, versus our approach of using time to treatment discontinuation [37-39].

The result of this study should be evaluated in context of a few limitations. Due to limited data availability from CheckMate 214, the impact of cabozantinib and lenvatinib + everolimus as second-line therapy after nivolumab + ipilimumab was based on the previously conducted NMA. It should be noted that the NMA was conducted for an intent-to-treat population, while the current analysis is restricted to patients with intermediate or poor risk. The analysis did not include sunitinib as a second-line treatment option as the clinical opinion noted sunitinib as more likely to be considered as a first-line treatment. Moreover, I-O-TKI combinations such as pembrolizumab + axitinib [26] and avelumab + axitinib [25] were not included in the analysis due to limited TFI and follow-up data from recently published clinical trials. Future research should focus on clinical and economic outcomes in these combinations, pending availability of long-term follow-up data and potentially randomized controlled trials comparing nivolumab + ipilimumab with such I-O-TKI combinations.

The analysis considered only two lines of active treatment, beyond which patients are assumed to receive best supportive care. However, given the estimated LYs after second line of 1.5-3 years, some proportion of patients 
could receive a third line of treatment. Since limited data on third-line treatment were available, we could not model three lines of treatment.

The assumption of equivalent efficacy was made for pazopanib and sunitinib. Only cost differences for drug acquisition and toxicity management were considered. This was based on findings from the COMPARZ trial, which did not report any statistically significant OS differences between sunitinib and pazopanib [27]. Patient-level cabozantinib clinical trial data were not available. Hence, NMA-based HR for time-to-treatment discontinuation on first-line cabozantinib versus sunitinib (HR: 0.66; 95\% CI: 0.46-0.95) and HR for OS on first-line cabozantinib versus sunitinib (HR: 0.80; 95\% CI: 0.50-1.27) were applied to model-simulated sunitinib curves for time to treatment discontinuation and OS, respectively [29]. It should be noted, however, that the NMA was based on the CABOSUN and CheckMate 214 trials, which have distinct differences in patient populations. Given the lack of head-to-head trial data, this was considered as a reasonable alternative to model efficacy of cabozantinib treatment. The impact of cabozantinib as first-line treatment on second-line treatment agents was not available. Given the similarity in mechanisms of action between sunitinib and cabozantinib, we assume that the impact on second-line treatments would be similar and any differences would be captured through the HR for cabozantinib versus sunitinib.

The impact of cabozantinib and lenvatinib + everolimus on OS when used as second-line therapy was not available. We used HRs from NMAs to model OS on second-line cabozantinib and lenvatinib + everolimus therapies [29]. A very recent study assessing the impact of cabozantinib after immunotherapy was associated with a median time to treatment failure of 6.6 months (95\% CI: 5.3-8.5 months). These results are consistent with our model predictions of second-line LYs of 0.64 years for the sequence of nivolumab + ipilimumab followed by cabozantinib [40].

Similarly, estimates for impact of pazopanib on OS as a second-line therapy were unreliable given that only 20 patients received sunitinib followed by pazopanib in CheckMate 214. Hence, we assumed that the impact of pazopanib on OS in the second line is equivalent to that of everolimus, and used the NMA of second-line aRCC treatments [29]. We also tested an alternative scenario in which the impact of pazopanib on OS in the second line was assumed to be equivalent to that of axitinib. Under this scenario, LYs increased from 4.75 to 5.8 years for the treatment sequence of nivolumab + ipilimumab followed by pazopanib, and the sequence continued to be cost-effective.

A maximum 2-year treatment duration for nivolumab + ipilimumab was assumed based on the latest revision in the CheckMate 214 study protocol and clinical opinion for cost calculations. This assumption was also in line with the treatment duration assumption made in previous submissions of I-Os such as nivolumab monotherapy as a second-line treatment for patients with aRCC [41] and pembrolizumab in pretreated patients with non-small-cell lung cancer [42] to the National Institute of Health and Care Excellence. However, it should be noted that patients were observed to remain on treatment beyond 2 years in CheckMate 214. When we tested a scenario with a maximum duration of 5 years, the prolonged treatment duration led to an increase in first-line treatment costs of $\$ 68,500$ versus the base case while maintaining the TFI and OS benefit. However, the total costs per LY for the nivolumab + ipilimumab treatment sequences (range: $\$ 59,678-\$ 86,488$ ) were still lower compared with the total cost per LY on other corresponding TKI-initiating sequences (range: $\$ 63,560-\$ 114,684$ ).

\section{Conclusion}

In patients with intermediate- or poor-risk aRCC, the use of nivolumab + ipilimumab followed by TKI monotherapies was associated with longer survival gains and was more cost-effective (in terms of cost per LY and cost per QALY gained) compared with treatment sequences with TKIs followed by nivolumab or cycling TKIs. The findings from scenario analyses and univariate sensitivity analyses were consistent with the base-case findings, even when the nivolumab + ipilimumab treatment duration was increased from a 2-year to a 5-year duration. Probabilistic sensitivity analyses confirmed that below the ICER threshold of $\$ 150,000$, nivolumab + ipilimumab followed by axitinib and nivolumab + ipilimumab followed by cabozantinib had the highest probability of being cost-effective among all treatment sequences. Clinical trials with head-to-head comparisons of treatment sequences are required to validate the findings of this study. 
Summary points

- In the past two decades, multiple immuno-oncology and targeted therapies have been approved for the treatment of advanced renal cell carcinoma in the first- and second-line setting. As new classes of drugs and combination treatments emerge, there is an interest to gain a deeper understanding of optimal treatment sequencing.

- A cost-effectiveness analysis was conducted to assess clinical and economic outcomes associated with various treatment sequences for patients with intermediate- or-poor risk advanced renal cell carcinoma. The treatment sequences included sunitinib, pazopanib, cabozantinib, and nivolumab + ipilimumab as first-line treatment, and axitinib, pazopanib, cabozantinib, nivolumab, and lenvatinib + everolimus as second-line treatment.

- A patient-level discrete event simulation was developed to evaluate cost and health outcomes over a patient's lifetime.

- Efficacy inputs were derived using patient-level data from Phase III clinical trials (CheckMate 214 and CheckMate 025) and a network meta-analysis study of published clinical trials. The multivariate risk equations derived for nivolumab + ipilimumab and sunitinib based on the CheckMate 214 trial accounted for relationships between individual patient characteristics and outcomes (treatment duration, treatment-free interval, best overall response and survival).

- Sequences initiating with nivolumab + ipilimumab resulted in the highest survival gain (mean 5.8-7.6 life-years and 3.6-5.3 quality-adjusted life-years [QALYs]) compared with sequences initiating with tyrosine kinase inhibitors (mean 3.1-5.5 life-years and 2.1-3.7 QALYs).

- Incremental cost-effectiveness ratio per QALY gained for nivolumab + ipilimumab initiating sequences were $\$ 16,524-\$ 125,860$ compared with the tyrosine kinase inhibitor-initiating sequences.

Supplementary data

To view the supplementary data that accompany this paper please visit the journal website at: www.futuremedicine.com/doi/suppl/10.2217/imt-2019-0199

Author contributions

A Ambavane, S Yang and S Rao contributed to the study conceptualization. S Yang and A Ambavane contributed to the methodology. A Shah is responsible for the formal analysis. All the authors performed investigation. All the authors are responsible for the writing of original draft, review and editing. All the authors contributed to and approved the manuscript.

Acknowledgments

Bristol-Myers Squibb (Princeton, NJ) and ONO Pharmaceutical Company Ltd (Osaka, Japan).

Financial \& competing interests disclosure

A Ambavane and A Shah are employees of Evidera, Inc., who were paid consultants to Bristol-Myers Squibb in relation to this study. S Yang and S Rao are employees of Bristol-Myers Squibb. MB Atkins reports consulting or advisory role with Bristol-Myers Squibb, Merck, Novartis, Arrowhead, Pfizer, Galactone, Werewolf, Fathom, Pneuma, Leads, Genentech-Roche, Exelixis, Eisai, Aveo, Array, AstraZeneca, Ideera, Aduro, ImmunoCore, Boehringer-Ingelheim, lovance, NewLink Pharma, Surface, Alexion, Acceleron, Cota and Amgen; stock ownership of Werewolf, Pyxis Oncology; research funding (institution) from Bristol-Myers Squibb, Merck, Pfizer and Genentech. MM Regan reports consultancy and travel fees from Merck and Bristol-Myers Squibb, and the following financial interests on behalf of her institution: research funding from Pfizer, Ipsen, Novartis, Merck, Ferring, AstraZeneca, Pierre Fabre, Roche, TerSera, Bristol-Myers Squibb, Astellas Pharma, Bayer, Medivation, Janssen Pharmaceuticals, Millennium Pharmaceuticals, Sanofi, Sotio and Dendreon, and consulting or advisory roles from Ipsen. DF McDermott reports consulting or advisory roles with Bristol-Myers Squibb, Merck, Genentech/Roche, Exelixis, Novartis, X4 Pharmaceuticals, Peloton Therapeutics, EMD Serono, Jounce Therapeutics, Alkermes and Lilly; research funding from Prometheus Laboratories, Bristol Myers-Squibb, Merck, Genentech, Novartis, Alkermes (institution) and Peloton Therapeutics (institution); and other relationships (Beth Israel Deaconess Medical Center, various). MD Michaelson reports consulting or advisory roles with Exelixis, Eisai, Novartis and Pfizer. The authors have no other relevant affiliations or financial involvement with any organization or entity with a financial interest in or financial conflict with the subject matter or materials discussed in the manuscript apart from those disclosed.

Writing and editorial assistance was provided by Caroline Cole of Evidera Inc, supported by Bristol-Myers Squibb. Editorial support was provided by Parexel, supported by Bristol-Myers Squibb. 
Data sharing statement

The authors certify that this manuscript reports the secondary analysis of clinical trial data that have been shared with them, and that the use of this shared data is in accordance with the terms (if any) agreed upon their receipt. The source of this data is: CheckMate 214 (NCT02231749) and CheckMate 025 (NCT01668784). Bristol-Myers Squibb's policy on data sharing may be found at www.bms.com/researchers-and-partners/independent-research/data-sharing-request-process.html

\section{Open access}

This work is licensed under the Attribution-NonCommercial-NoDerivatives 4.0 Unported License. To view a copy of this license, visit http://creativecommons.org/licenses/by-nc-nd/4.0/

\section{References}

Papers of special note have been highlighted as: $\bullet$ of interest; $\bullet \bullet$ of considerable interest

1. American Cancer Society. Cancer facts \& figures (2017).

www.cancer.org/research/cancer-facts-statistics/all-cancer-facts-figures/cancer-facts-figures-2017.html

2. Gangadaran SGD. Current management options in metastatic renal cell cancer. Oncol. Rev. 11(2), 339 (2017).

3. Howlader N, Noone AM, Krapcho M et al. SEER cancer statistics review (CSR), 1975-2016, National Cancer Institute. https://seer.cancer.gov/csr/1975_2016

4. Heng DY, Xie W, Regan MM et al. Prognostic factors for overall survival in patients with metastatic renal cell carcinoma treated with vascular endothelial growth factor-targeted agents: results from a large, multicenter study. J. Clin. Oncol. 27(34), 5794-5799 (2009).

5. Heng DY, Xie W, Regan MM et al. External validation and comparison with other models of the International Metastatic Renal-Cell Carcinoma Database Consortium prognostic model: a population-based study. Lancet Oncol. 14(2), 141-148 (2013).

6. Tamada S, Iguchi T, Yasuda S, Kato M, Yamasaki T, Nakatani T. The difference in the survival rate of patients with metastatic renal cell carcinoma in the intermediate-risk group of the Memorial Sloan Kettering Cancer Center criteria. Oncotarget 9(45), 27752-27759 (2018).

7. National Comprehensive Cancer Network. NCCN clinical practice guidelines in oncology, kidney cancer. Version 2. www.nccn.org/professionals/physician_gls/pdf/kidney.pdf

8. Atkins MB, Tannir NM. Current and emerging therapies for first-line treatment of metastatic clear cell renal cell carcinoma. Cancer Treat. Rev. 70, 127-137 (2018).

9. Fischer S, Gillessen S, Rothermundt C. Sequence of treatment in locally advanced and metastatic renal cell carcinoma. Transl. Androl. Urol. 4(3), 310-325 (2015).

10. Motzer RJ, Escudier B, McDermott DF et al. Nivolumab versus everolimus in advanced renal-cell carcinoma. N. Engl. J. Med. 373(19), 1803-1813 (2015).

-. The Phase III CheckMate 025 study demonstrated that among patients with previously treated advanced renal-cell carcinoma (aRCC), overall survival was longer with nivolumab compared with everolimus.

11. Motzer RJ, Tannir NM, McDermott DF et al. Nivolumab plus ipilimumab versus sunitinib in advanced renal-cell carcinoma. N. Engl. J. Med. 378(14), 1277-1290 (2018).

-. The Phase III CheckMate 214 study demonstrated that among intermediate- and poor-risk patients with previously untreated aRCC, overall survival and objective response rates were significantly higher with nivolumab plus ipilimumab than with sunitinib.

12. Postow MA, Chesney J, Pavlick AC et al. Nivolumab and ipilimumab versus ipilimumab in untreated melanoma. N. Engl. J. Med. 372(21), 2006-2017 (2015).

13. Schadendorf D, Wolchok JD, Hodi FS et al. Efficacy and safety outcomes in patients with advanced melanoma who discontinued treatment with nivolumab and ipilimumab because of adverse events: A pooled analysis of randomized Phase II and III trials. J. Clin. Oncol. 35(34), 3807-3814 (2017).

14. Ornstein MC, Wood LS, Hobbs BP et al. A Phase II trial of intermittent nivolumab in patients with metastatic renal cell carcinoma (mRCC) who have received prior anti-angiogenic therapy. J Immunother. Cancer 7(1), 127 (2019).

15. Takagi T, Yoshida K, Kobayashi $\mathrm{H}$ et al. Durable response after discontinuation of nivolumab therapy in patients with metastatic renal cell carcinoma. Jpn. J. Clin. Oncol. 48(9), 860-863 (2018).

16. McDermott DF, Rini BI, Motzer RJ et al. Treatment-free interval (TFI) following discontinuation of first-line nivolumab plus ipilimumab $(\mathrm{N}+\mathrm{I})$ or sunitinib $(\mathrm{S})$ in patients (Pts) with advanced renal cell carcinoma (aRCC): checkMate 214 analysis. Ann. Oncol. 29(Suppl. 8), 874P (2018).

- The post hoc analysis of the CheckMate 214 trial showed that nivolumab + ipilimumab was associated with significantly longer treatment-free survival beyond treatment discontinuation in patients with intermediate- or poor-risk aRCC irrespective of whether patients achieved response or disease control. 
17. Regan MM, Atkins MB, Powles T et al. Treatment-free survival, with and without toxicity, as a novel outcome applied to immuno-oncology agents in advanced renal cell carcinoma. Ann. Oncol. 30(Suppl. 8), 971P (2019).

18. Kreamer KM. Immune checkpoint blockade: A new paradigm in treating advanced cancer. J. Adv. Pract. Oncol. 5(6), 418-431 (2014).

19. Hoos A, Ibrahim R, Korman A et al. Development of ipilimumab: contribution to a new paradigm for cancer immunotherapy. Semin. Oncol. 37(5), 533-546 (2010).

20. Saenger YM, Wolchok JD. The heterogeneity of the kinetics of response to ipilimumab in metastatic melanoma: patient cases. Cancer Immun. 8(1), (2008).

21. Wolchok JD, Hoos A, O'Day $S$ et al. Guidelines for the evaluation of immune therapy activity in solid tumors: immune-related response criteria. Clin. Cancer Res. 15(23), 7412-7420 (2009).

22. Richardson P, Roy A, Acharyya $S$ et al. Treatment-free interval as a metric of patient experience and a health outcome of value for advanced multiple myeloma: the case for the histone deacetylase inhibitor panobinostat, a next-generation novel agent. Expert Rev. Hematol. 10(10), 933-939 (2017).

23. Acaster S, Gaugris S, Velikova G, Yong K, Lloyd AJ. Impact of the treatment-free interval on health-related quality of life in patients with multiple myeloma: a UK cross-sectional survey. Support. Care Cancer 21(2), 599-607 (2013).

24. Tarhini A, McDermott D, Benedict A et al. Quantifying the treatment-free interval after first-line immuno-oncology (IO) treatment of advanced melanoma. Presented at: 9th World Congress of Melanoma and the 14th International Congress of the Society for Melanoma Research. Brisbane, Australia, 18-21 October 2017.

25. Motzer RJ, Penkov K, Haanen J et al. Avelumab plus axitinib versus sunitinib for advanced renal-cell carcinoma. N. Engl. J. Med. 380(12), 1103-1115 (2019).

26. Rini BI, Plimack ER, Stus V et al. Pembrolizumab plus axitinib versus sunitinib for advanced renal-cell carcinoma. N. Engl. J. Med. 380(12), 1116-1127 (2019).

27. Motzer RJ, Hutson TE, Cella D et al. Pazopanib versus sunitinib in metastatic renal-cell carcinoma. N. Engl. J. Med. 369(8), 722-731 (2013).

- The Phase III COMPARZ trial demonstrated that pazopanib had similar efficacy to that of sunitinib when used as a first-line therapy in patients with metastatic renal-cell carcinoma.

28. Bristol-Myers Squibb-Evidera. CheckMate 214. Statistical Analysis 2018. Data on File. (2018).

29. Bristol-Myers Squibb-Parexel. Systematic review (SR) in RCC, Version 3. (2016).

30. Bristol-Myers Squibb-Amaris. Network meta-analysis (NMA) of clinical data in previously untreated, advanced or metastatic renal cell carcinoma. Study report (Internal report). (2018).

31. Centers for Disease Control and Prevention. United States Life Tables 2014 and Statistical Analysis 2018. Data on File. www.cdc.gov/nchs/products/life_tables.htm

32. United States Department of Labor. Consumer price index. www.bls.gov/cpi/detailed-report.htm

33. National Institute for Health and Care Excellence. Lenvatinib with everolimus for previously treated advanced renal cell carcinoma. Technology appraisal guidance [TA498]. www.nice.org.uk/guidance/ta498

34. Swinburn P, Lloyd A, Nathan P, Choueiri TK, Cella D, Neary MP. Elicitation of health state utilities in metastatic renal cell carcinoma. Curr. Med. Res. Opin. 26(5), 1091-1096 (2010).

35. Bristol-Myers Squibb. CheckMate 214 - Final Clinical Study Report (CSR) - CA209214. BMS-936558. 2018. (2018).

36. Briggs AH, Weinstein MC, Fenwick EA et al. Model parameter estimation and uncertainty analysis: a report of the ISPOR-SMDM Modeling Good Research Practices Task Force Working Group-6. Med. Decis. Making 32(5), 722-732 (2012).

37. Wan X, Zhang Y, Tan C, Zeng X, Peng L. First-line nivolumab plus ipilimumab vs sunitinib for metastatic renal cell carcinoma: a cost-effectiveness analysis. JAMA Oncol. 5(4), 491-496 (2019).

38. Wu B, Zhang Q, Sun J. Cost-effectiveness of nivolumab plus ipilimumab as first-line therapy in advanced renal-cell carcinoma. J. Immunother. Cancer 6(1), 124 (2018).

39. Reinhorn D, Sarfaty M, Leshno $M$ et al. A cost-effectiveness analysis of nivolumab and ipilimumab versus sunitinib in first-line intermediate- to poor-risk advanced renal cell carcinoma. Oncologist 24(3), 366-371 (2019).

40. McGregor BA, Lalani A, Xie W et al. Activity of cabozantinib (cabo) after PD-1/PD-L1 immune checkpoint blockade (ICB) in metastatic clear cell renal cell carcinoma (mccRCC). Ann. Oncol. 29(Suppl. 8), 879P (2018).

41. National Institute for Health and Care Excellence. Technology appraisal guidance [TA417]. www.nice.org.uk/guidance/ta417

42. National Institute for Health and Care Excellence. Pembrolizumab for treating PD-L1-positive non-small-cell lung cancer after chemotherapy. Technology appraisal guidance [TA428]. www.nice.org.uk/guidance/ta428. 